\title{
Ordres bipartitionnaires et statistiques sur les mots
}

\author{
Guo-Niu HAN $(*)$
}

Dédié à Dominique Foata à l'occasion de son soixantième anniversaire

Submitted : February 9, 1995; Accepted : March 28, 1995

RÉSumÉ. - Soit $U$ un ensemble de couples de lettres. Foata et Zeilberger [FZ] ont introduit les $U$ statistiques pour les mots quelconques. Dans cette note, on établit une condition nécessaire et suffisante pour que les deux définitions "maj $j_{U}$ " et "maj $2_{U}$ ", qu'on rencontre dans le cas classique $[\mathrm{H}]$, sont équivalentes. Il est remarquable que cette condition est exactement la même que celle qui a été trouvée pour l'équidistribution des deux statistiques " $\operatorname{maj}_{U}$ " et "inv $U$ ".

1. Introduction. - Soient $X$ un alphabet et $X^{*}$ le monoïde libre engendré par $X$. Si $w=x_{1} x_{2} \cdots x_{m} \in X^{*}$ est un mot de longueur $m$, on appelle réarrangement de $w$ tout mot $u=x_{\sigma_{1}} x_{\sigma_{2}} \cdots x_{\sigma_{m}}$, où $\sigma$ est une permutation appartenant à $\mathfrak{S}_{m}$. On note $R(w)$ la classe de tous les réarrangements du mot $w$.

On représente les sous-ensembles $U \subset X \times X$ comme suit : pour tout $x, y \in X$, on écrit $x \succ y$ si $(x, y) \in U$ et $x \nsucc y$ si $(x, y) \notin U$. Un sous-ensemble $U$ est appelé ordre bipartitionnaire, $\left({ }^{* *}\right)$ si les deux conditions suivantes sont remplies :

$$
\begin{aligned}
& z \succ y \text { et } y \succ x \Longrightarrow z \succ x ; \\
& z \succ y \text { et } x \nsucc y \Longrightarrow z \succ x .
\end{aligned}
$$

Pour une relation $U \subset X \times X$ quelconque, on généralise les statistiques classiques "inv" (nombre d'inversions) et "maj" (indice majeur) pour les mots quelconques de la façon suivante. Soit $w=x_{1} x_{2} \cdots x_{m} \in X^{*}$ un mot, on définit :

$$
\begin{gathered}
\operatorname{inv}_{U} w=\operatorname{Card}\left\{(i, j) \mid 1 \leq i<j \leq m, x_{i} \succ x_{j}\right\} \\
\operatorname{maj}_{U} w=\sum\left\{i \mid 1 \leq i \leq n-1, x_{i} \succ x_{i+1}\right\} .
\end{gathered}
$$

Lorsque $X$ est totalement ordonné, l'ordre sous-jacent " $>$ " vérifie bien les deux conditions (1) et (2), et est donc bipartitionnaire. Lorsque le sous-ensemble $U$ est cet ordre, c'est-à-dire, $x \succ y$ si et seulement si $x>y$, on a alors $\operatorname{inv}_{U}=$ inv et $\operatorname{maj}_{U}=$ maj. De plus, on peut vérifier que les deux statistiques précédentes généralisent les statistiques inv $_{k}$ et $\mathrm{maj}_{k}$ introduites dans [CF2]. Un autre exemple d'ordre bipartitionnaire se trouve dans

$\left(^{*}\right)$ Avec le concours du programme des Communautés Européennes en Combinatoire Algébrique, 1994-95.

$\left.{ }^{* *}\right)$ Cette définition est différente de celle qui a été donnée dans [FZ]. Le théorème 5 dans la dernière section montre que ces deux définitions sont équivalentes. 
la section 3. Le nombre des ordres bipartitionnaires $B_{r}$ sur un alphabet de cardinal $r$ est donné par la fonction génératrice suivante [FZ] :

$$
\begin{aligned}
\sum_{r \geq 0} B_{r} \frac{u^{r}}{r !} & =\frac{1}{3-2 e^{u}} \\
& =1+2 u+10 \frac{u^{2}}{2 !}+74 \frac{u^{3}}{3 !}+730 \frac{u^{4}}{4 !}+9002 \frac{u^{5}}{5 !}+\cdots
\end{aligned}
$$

Foata et Zeilberger ont établi le résultat suivant [FZ] :

Théorème 1 (Fonta et Zeilberger). - Les deux statistiques maj met $_{U}$ inv sont équidistribuées, si et seulement si le sous-ensemble $U$ est un ordre bipartitionnaire.

Depuis $[\mathrm{H}]$, on sait qu'il existe une autre définition "maj2 $2_{U}$ " qui est équivalente à "maj" dans le cas classique. Cette statistique peut être aussi généralisée pour un sous-ensemble $U \in X \times X$ quelconque. La définition de "maj2 $U$ " se trouve dans la section 2 . Dans cette note, on démontre le théorème suivant :

ThÉorème 2. - Les deux statistiques maj $_{U}$ et maj2 ${ }_{U}$ sont équivalentes, si et seulement si le sous-ensemble $U$ est un ordre bipartitionnaire.

D'après les deux théorèmes précédents, on peut dire que les ordres bipartitionnaires sont des objets combinatoires "naturels".

2. Intervalles cycliques. - Pour $x, y \in X$, l'intervalle cyclique (généralisé), noté $\rrbracket x, y \rrbracket_{U}$, est défini par

$$
\rrbracket x, y \rrbracket_{U}= \begin{cases}\{z \in X \mid z \succ x \text { et } z \nsucc y\}, & \text { si } x \nsucc y ; \\ \{z \in X \mid z \succ x \text { ou } z \nsucc y\}, & \text { si } x \succ y .\end{cases}
$$

Parfois, on a besoin d'ajouter l'élément " $\infty$ " dans notre alphabet pour former l'ensemble $[r] \cup\{\infty\}$, et on suppose alors $x \nsucc \infty$ et $\infty \succ x$ pour tout $x$. Dans ce cas, on a $z \in \rrbracket x, \infty \rrbracket_{U}$ si et seulement si $z \succ x$. Deux exemples d' intervalle cyclique se trouvent dans la section 3 .

Soient $w=x_{1} x_{2} \cdots x_{m} \in X^{*}$ un mot et $S \subset X$, on note

$$
\langle w, S\rangle=\#\left\{i \mid 1 \leq i \leq m, x_{i} \in S\right\}
$$

Soient $w=x_{1} x_{2} \cdots x_{m}, u \in X^{*}$ deux mots et $S, T \in X$ deux sous-ensembles. On a les propriétés suivantes :

$$
\begin{aligned}
\langle w, \emptyset\rangle & =0,\langle w, X\rangle=m, \\
\langle w u, S\rangle & =\langle w, S\rangle+\langle u, S\rangle \\
\langle w, S\rangle+\langle w, T\rangle & =\langle w, S \cup T\rangle+\langle w, S \cap T\rangle .
\end{aligned}
$$


THE ELECTRonic JoURnal of COMBinatorics 3 (2) (1996), \#R3

Définition. - Soient $w=x_{1} x_{2} \cdots x_{m}$ un mot. Avec la convention $x_{n+1}=\infty$, on définit :

$$
\operatorname{maj} 2_{U} w=\sum_{j=1}^{m}\left\langle x_{1} x_{2} \cdots x_{j-1}, \rrbracket x_{j}, x_{j+1} \rrbracket_{U}\right\rangle .
$$

La suite $\left(s_{j}\right)_{1 \leq j \leq m}$ où $s_{j}=\left\langle x_{1} x_{2} \cdots x_{j-1}, \rrbracket x_{j}, x_{j+1} \rrbracket_{U}\right\rangle$ est appelée maj-codage du mot $w$.

3. Exemple. - Considérons l'alphabet $X=\{0,1,2, \cdots, 6\}$. On vérifie que l'ensemble

$$
U=\left\{\begin{array}{llll}
0 \succ 0, & 0 \succ 1, & & \\
1 \succ 0, & 1 \succ 1, & & \\
2 \succ 0, & 2 \succ 1, \quad 2 \succ 2, & \\
3 \succ 0, & 3 \succ 1, \quad 3 \succ 2, & \\
4 \succ 0, & 4 \succ 1, \quad 4 \succ 2, & \\
5 \succ 0, & 5 \succ 1, \quad 5 \succ 2, & \\
6 \succ 0, & 6 \succ 1, \quad 6 \succ 2, \quad 6 \succ 3, \quad 6 \succ 4, \quad 6 \succ 5
\end{array}\right\}
$$

est bien un ordre bipartitionnaire. On a par exemple les intevalles cycliques \6,2 $\rrbracket_{U}=\{0,1\}$ et $\rrbracket 2,3 \rrbracket_{U}=\rrbracket 2,4 \rrbracket_{U}=\rrbracket 2,5 \rrbracket_{U}=\{2,3,4,5\}$. Pour le mot $w=315016406254320$, on a $\operatorname{maj}_{U} w=1+3+4+6+7+9+13+14=57$ et maj $2_{U} w=1+3+4+5+1+6+5+3+6+9+14=$ 57. Les calculs complets sont consignés dans le tableau suivant :

$\begin{array}{cccccccccccccccc}i & 1 & 2 & 3 & 4 & 5 & 6 & 7 & 8 & 9 & 10 & 11 & 12 & 13 & 14 & 15 \\ x_{i} & 3 & 1 & 5 & 0 & 1 & 6 & 4 & 0 & 6 & 2 & 5 & 4 & 3 & 2 & 0 \\ \text { descente } & - & & - & - & & - & - & & - & & & & - & - & \\ s_{i} & 0 & 1 & 0 & 3 & 4 & 5 & 1 & 7 & 4 & 3 & 0 & 0 & 6 & 9 & 14\end{array}$

4. Propriétés des intervalles cycliques. - Soit $U \in X \times X$ un sous-ensemble. Pour abréger les écritures, notons $A=\rrbracket y, \infty \rrbracket_{U}, B=\rrbracket x, y \rrbracket_{U}, C=\rrbracket x, \infty \rrbracket_{U}$ pour deux lettres $x, y \in X$. On a le résultat suivant :

Lemme 3. - Le sous-ensemble $U$ est un ordre bipartitionnaire, si et seulement si, pour tout $x, y \in X$, les relations entre $A, B$ et $C$ suivants sont remplies :

(i) si $x \nsucc y$, alors $A \cap B=\emptyset, A \cup B=C$;

(ii) si $x \succ y$, alors $A \cap B=C, A \cup B=X$.

Démonstration. - La partie "si". — D'après la condition (i), pour tout $x, y$, si $x \nsucc y$ et $z \in A$, alors $z \in C$. Ceci démontre la relation (1) de la section 1. D'après la condition (ii), pour tout $x, y$, si $x \succ y$ et $z \in C$, alors $z \in A$. Ceci démontre la relation (2) de la section 1 .

La partie "seulement si". — Dans le premier cas, i.e., $x \nsucc y$, on vérifie :

- Si $z \in C$, alors $z \succ x$. Dans ce cas, on a ou bien $z \succ y, z \in A$ et $z \notin B$; ou bien $z \nsucc y, z \in B$ et $z \notin A$; 
- Si $z \in A$, alors $z \succ y$. D'après la relation (1), on a $z \succ x, z \in C$;

- Si $z \in B$, alors $z \succ x$ et $z \nsucc y$. On a $z \in C$.

Dans le second cas, i.e., $x \succ y$, on vérifie :

- Si $z \in C$, alors $z \succ x, z \in B$. D'autre part, d'après (2), on a $z \succ y$ et $z \in A$.

- Si $z \notin C$, i.e., $z \nsucc x$. Dans ce cas, si $z \succ y$, alors $z \in A$ et $z \notin B$; si $z \nsucc y$, alors $z \notin A$ et $z \in B$.

5. Démonstration du théorème 2. - Posons $w^{\prime}=x_{1} x_{2} \cdots x_{m-1}$. On note $\left(s_{j}\right)_{1 \leq j<m}$ et $\left(s_{j}^{\prime}\right)_{1 \leq j<m-1}$ les maj-codages de $w$ et de $w^{\prime}$ respectivement, il est clair qu'on a $s_{j}=s_{j}^{\prime}$ pour tout $j<m-1$. Pour abréger les écritures, notons $A=\rrbracket x_{m}, \infty \rrbracket_{U}, B=$ $\rrbracket x_{m-1}, x_{m} \rrbracket_{U}, C=\rrbracket x_{m-1}, \infty \rrbracket_{U}$ et $u=x_{1} x_{2} \cdots x_{m-2}$. On calcule maintenant

$$
\begin{aligned}
\Delta & =s_{m}+s_{m-1}-s_{m-1}^{\prime} \\
& =\left\langle u x_{m-1}, A\right\rangle+\langle u, B\rangle-\langle u, C\rangle \\
& =\left\langle x_{m-1}, A\right\rangle+\langle u, A \cup B\rangle+\langle u, A \cap B\rangle-\langle u, C\rangle .
\end{aligned}
$$

La partie "si". - Si $x_{m-1} \nsucc x_{m}$, alors $x_{m-1} \notin A$. D'après les propriétés précédentes, on a

$$
\Delta=0+\langle u, C\rangle+\langle u, \emptyset\rangle-\langle u, C\rangle=0 .
$$

D'autre part, si $x_{m-1} \succ x_{m}$, alors $x_{m-1} \in A$, on a

$$
\Delta=1+\langle u, X\rangle+\langle u, C\rangle-\langle u, C\rangle=m-1 .
$$

D'où $\operatorname{maj}_{U} w=\operatorname{maj}_{U} w$ par récurrence sur la longueur $m$ du mot $w$.

La partie "seulement si". - Puisque $\operatorname{maj}_{U} w=\operatorname{maj}_{U} w$ pour tout $w$, on a $\Delta=0$ si $x_{m-1} \nsucc x_{m}$ et $\Delta=m-1$ si $x_{m-1} \succ x_{m}$. Dans le premier cas, on a $\Delta=$ $0+\langle u, A \cup B\rangle+\langle u, A \cap B\rangle-\langle u, C\rangle=0$ pour tout $u$ de longueur $m-2$, et forcément $A \cap B=\emptyset$ et $A \cup B=C$; et dans le second cas, on a $\Delta=1+\langle u, A \cup B\rangle+\langle u, A \cap B\rangle-\langle u, C\rangle=m-1$ pour tout $u$ de longueur $m-2$, et forcément $A \cap B=C$ et $A \cup B=X$. D'après le lemme précédent, le sous-ensemble $U$ est donc un ordre bipartitionnaire.

6. Structures des ordres bipartitionnaires. - D'après la définition de l'ordre bipartitionnaire, on a immédiatement les propriétés suivantes :

$$
\begin{aligned}
& x \nsucc y \text { et } z \succ y \Longrightarrow x \nsucc z ; \\
& x \nsucc y \text { et } y \nsucc z \Longrightarrow x \nsucc z ; \\
& x \succ y \text { et } y \succ x \Longrightarrow x \succ x \text { et } y \succ y ; \\
& x \nsucc y \text { et } y \nsucc x \Longrightarrow x \nsucc x \text { et } y \nsucc y .
\end{aligned}
$$

Soit $U \subset X \times X$ un sous-ensemble quelconque. Pour tout $x, y \in X$, on dit que la lettre $x$ est incomparable à $y$, si $x \succ y$ et $y \succ x$ ou bien si $x \nsucc y$ et $y \nsucc x$. On note $x \approx y$ si $x$ est incomparable à $y$, et la relation " $\approx$ " est appelée relation d'incomparabilité définie par $U$. 
THE ELECTRONIC JOURNAL OF COMBINATORICs 3 (2) (1996), \#R3

Lemme 4. - Si $U \subset X \times X$ est un ordre bipartitionnaire, alors la relation d'incomparabilité " $\approx$ " définie par $U$ est une relation d'équivalence.

Démonstration. - La reflexivité et la symétrie sont évidentes d'après la définition. Pour la transitivité, on suppose $x \approx y$ et $y \approx z$. D'abord, si $x \succ y$ et $y \succ x$, on a $y \succ y$ d'après (5). On a alors $y \succ z$ et $z \succ y$. Ainsi, par la relation (1), on a $x \approx z$. Dans le second cas, si $x \nsucc y$ et $y \nsucc x$, c'est la même vérification.

DÉfinition. - Une bipartition ordonnée de $X$ est une suite ordonnée $\left(B_{1}, B_{2}, \cdots, B_{k}\right)$ de blocs non vides, disjoints deux à deux, de reunion $X$, dont certains peuvent être soulignés.

ThÉORÈme 5. - Une relation $U$ est bipartitionnaire, si et seulement s'il existe une bipartition ordonnée $B=\left(B_{1}, B_{2}, \cdots, B_{k}\right)$ de $X$, tel que

$$
y \succ x \Longleftrightarrow \begin{gathered}
x \in B_{l}, y \in B_{h} \text {, avec } l<h ; \\
\text { ou bien } x, y \in B_{l} \text {, avec } B_{l} \text { souligné. }
\end{gathered}
$$

Démonstration. — La partie "si" est triviale : il est suffit de vérifier les deux relations (1) et (2) de la section 1 . Pour la partie "seulement si", d'après le lemme précédent, l'ensemble quotient $X / \approx$ de $X$ par rapport à la relation d'incomparabilité " $\approx$ " existe. Il est clair qu'avec l'ordre induit par la relation $U$, cet ensemble $X / \approx$ est totalement ordonné. Soient $B_{1}, B_{2}, \cdots, B_{k}$ les éléments de $X / \approx$ suivant cet ordre total. On peut vérifier la relation (7) avec la suite de $B_{1}, B_{2}, \cdots, B_{k}$ ainsi définie.

Exemple. - Pour la relation $U$ donnée dans la section 3, la bipartition associée est $B=(\{\underline{0}, \underline{1}\},\{\underline{2}\},\{3,4,5\},\{6\})$.

\section{BIBLIOGRAPHIE}

[CF1] R. J. Clarke et D. FoAta. - Eulerian Calculus, I : Univariable Statistics, Europ. J. Combinatorics, t. 15, 1994, p. 345-362.

[CF2] R. J. Clarke et D. Foata. - Eulerian calculus, II : An extension of Han's fundamental transformation, à paraître dans Europ. J. Combinatorics.

[FZ] D. Foata et D. Zeilberger. - Graphical Major Indices, soumis pour publication, 1995.

$[\mathrm{K}]$ K. W. J. KADELL. - Weighted inversion numbers, restricted growth function, and standard Young tableaux, J. Comb. Theory, Ser. A, t. 40, 1985, p. 22-44.

[H] G.-N. HAN. - Une transformation fondamentale sur les réarrangements de mots, Advances in Math., t. 105, 1994, p. 26-41.

I.R.M.A., Université Louis Pasteur \& C.N.R.S. 7, rue René Descartes, 67084 Strasbourg Cedex, France email : guoniu@math.u-strasbg.fr 